\title{
DAMPING OF HIGHER-ORDER MODES IN A THREEFOLD SYMMETRY ACCELERATING STRUCTURE
}

\author{
D. $\mathrm{Yu}^{\circ}$ \\ DULY Consultants, Rancho Palos Verdes, CA 90732 \\ N. Kroll ${ }^{\dagger}$ \\ University of California, San Diego and SLAC
}

\begin{abstract}
We investigate a waveguide-coupled damping structure with threefold symmetry using the 2-D and 3-D MAFLA codes. Within the frequency range considered, all higher order modes except the TMO11 and TE111 modes are heavily damped. Possible ways to detrap these by using asymmetric waveguides offset with respect to the acceleratIng cavity in the direction of the beam are studicd. External $Q$ s and resonant frequencies are calculated using recently developed compulet methods.
\end{abstract}

\section{INTRODUCTION}

Much work has been done since the first workshop on Future Lincar Collider held in SLAC in 1988 on the design of damped accelerating structures using various waveguide loaded cavities. Accelerating structures in which higher order modes (HOM) are damped by coupling to waveguides via radial and circumferential slots have been roposed by R. Palmer ${ }^{1}$. We have studied several of these damped cavitics with radial or circumferential couplers and have calculated, in each instance, the extcrnal $Q$ s and resonant frequencies of the damped structure using a theory developed by Kroll and $\mathrm{Yu}^{2}$, based on frequencies and phase shifts for shorted waveguides. A useful extension and variation of this theory has been recently devel. oped $^{3}$ and is also used in our evaluation.

We begin by recapitulating some results for cavities with iwolold-symmetry radial or circum[erential slots which have been reported previously4. The calculated $Q$ for the principal transverse mode of the radially slotted cavities is between 8.7 to 13.2, depending on the waveguide cutorf frequency. Resuls for the $Q$ values using the Kroll-Yu method are highly accurate and convincing, and compare favorably with those oblained with other methods. In addition, the Kroll-Yu method determines the resonance frequencies accurately. A problem with the radial slot cavity is the existence of a slot mode which persistently has a lower frequency than the accelerating mode. Because it has a different symmetry from the accelerating mode, however, it can be strongly coupled out by use of a double ridge waveguide ${ }^{2}$ having a lower cutoft frequency than the fundamental mode, without significantly damping the latter.

An advantage of circumferentially coupled cavities is the absence of any slot modes. Not restricted by clcarance considerations, use of smaller irises in these cavities also helps preserve accelerating field structure and depress HOM longitudinal coupling. A potential problem of these cavities is that the lowest Iransverse frequency is very near the waveguide culoff. It is important to design the struc. ture with extreme care in order to avoid trapped transverse modes. The possibility exists that a transverse mode is irapped even when its frequency, in the absence of damping waveguides, is above the waveguide cutoft. Ideally, the waveguide cutoff firequency should be far enough below the HOM to avoid trapping, and yet be sufficiently high above the fundamental, so that the accelerating mode is well preserveds.

Recently, Arcioni and Conciauro ${ }^{6}$ (AC) proposed a threcfold symmetric, circumferentially coupled, waveguide loaded cavily. They showed that the shunt impedance and the $Q$ of the accelerating mode are degraded by $20 \%$ and $12 \%$, respectively, from those of a closed cavity, while most of the HOMs were effectively damped. In this research we applied our computer method to further analyze this Sband cavily. The resonance frequencies and external Qs of the HOM for this structure were calculated. Then we studied a similar X-band waveguide loaded accelerating structure with a threefold symmetry, and showed that in the frequency range considered, two HOMs (TMOLI and TE111) remain trapped. Finally, we studied methods to detrap these modes.

\section{FOLD SYMMETRY CAVITY}

The AC cavity shown in Figure 1 has a radius $(f)$ of $3.0 \mathrm{~cm}$ and a height of $3.5 \mathrm{~cm}$. The cylindrical cavity is coupled 10 three square waveguides, $120^{\circ}$ apart, each having a width of $3.5 \mathrm{~cm}$. We calculated the frequencies of
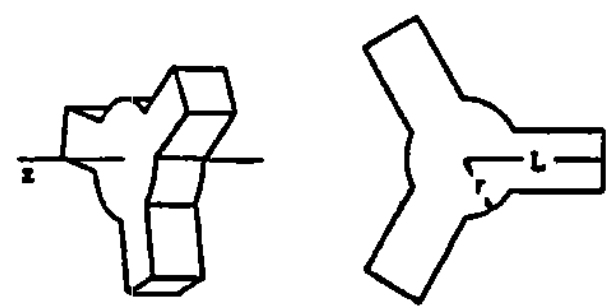

Fig. 1 A threcfold symmetry cavity (rel. 6)

the TM and TE modes of this structure with URMELT for waveguide lengths $(\mathrm{L})$ of $7.3,7.9,8.5,9.1$ and $18.0 \mathrm{~cm}$. For case of mode identification, only half of the structure was 
modeled with reflection symmetry imposed on the half plane. A 32000-point mesh is used for the half model with the longest guide length. The number of mesh points for shorter guide lengths was reduced to maintain compatible mesh fincncss. The beam pipc was not Included in the 2.D model as its effect on the external $Q$ was judged to be ncgligible. Table 1 lists the frequencies of the TM modes, with principal electric ficlds a long the axis of the resonator, shoricd at various guide lengths:

Table I Modal frequencies vs guide lengths for AC cavity

\begin{tabular}{|c|c|c|c|c|c|}
\hline$\frac{\text { Mode }}{\text { TMO10 }}$ & $\frac{7.3 \mathrm{~cm}}{3354.30}$ & $\frac{7.9 \mathrm{~cm}}{3360.94}$ & $\frac{8.5 \mathrm{~cm}}{3352.05}$ & $\frac{9.1 \mathrm{~cm}}{3356.40}$ & $\frac{18.0 \mathrm{~cm}}{3341.75}$ \\
\hline $\begin{array}{l}T M 110 \\
T M 020\end{array}$ & $\begin{array}{l}4637.98 \\
5104.74\end{array}$ & $\begin{array}{l}4603.07 \\
4989.96\end{array}$ & $\begin{array}{l}4556.38 \\
4865.69\end{array}$ & $\begin{array}{l}4526.22 \\
4785.28\end{array}$ & $\begin{array}{l}4335.70 \\
4376.92\end{array}$ \\
\hline $\begin{array}{l}\mathrm{TM} 120 \\
\mathrm{TM} 030\end{array}$ & $\begin{array}{l}5699.26 \\
6682.62\end{array}$ & $\begin{array}{l}5529.12 \\
6367.92\end{array}$ & $\begin{array}{l}5374.89 \\
6101.79\end{array}$ & $\begin{array}{l}525220 \\
5883.56\end{array}$ & $\begin{array}{l}4553.26 \\
4661.38\end{array}$ \\
\hline $\begin{array}{l}T M 130 \\
T M 210\end{array}$ & $\begin{array}{l}6955.29 \\
7956.85\end{array}$ & $\begin{array}{l}6730.32 \\
7683.32\end{array}$ & $\begin{array}{l}6498.38 \\
7448.07\end{array}$ & $\begin{array}{l}6292.62 \\
7255.65\end{array}$ & 4893.36 \\
\hline $\begin{array}{l}\text { TMO4O } \\
\text { TM140 } \\
\text { TMOSO } \\
\text { TM1SO }\end{array}$ & 8371.52 & 7927.96 & 7545.96 & $\begin{array}{l}7203.79 \\
8161.31 \\
8512.02\end{array}$ & $\begin{array}{l}5101.62 \\
5340.50 \\
5640.47 \\
5865.92\end{array}$ \\
\hline
\end{tabular}
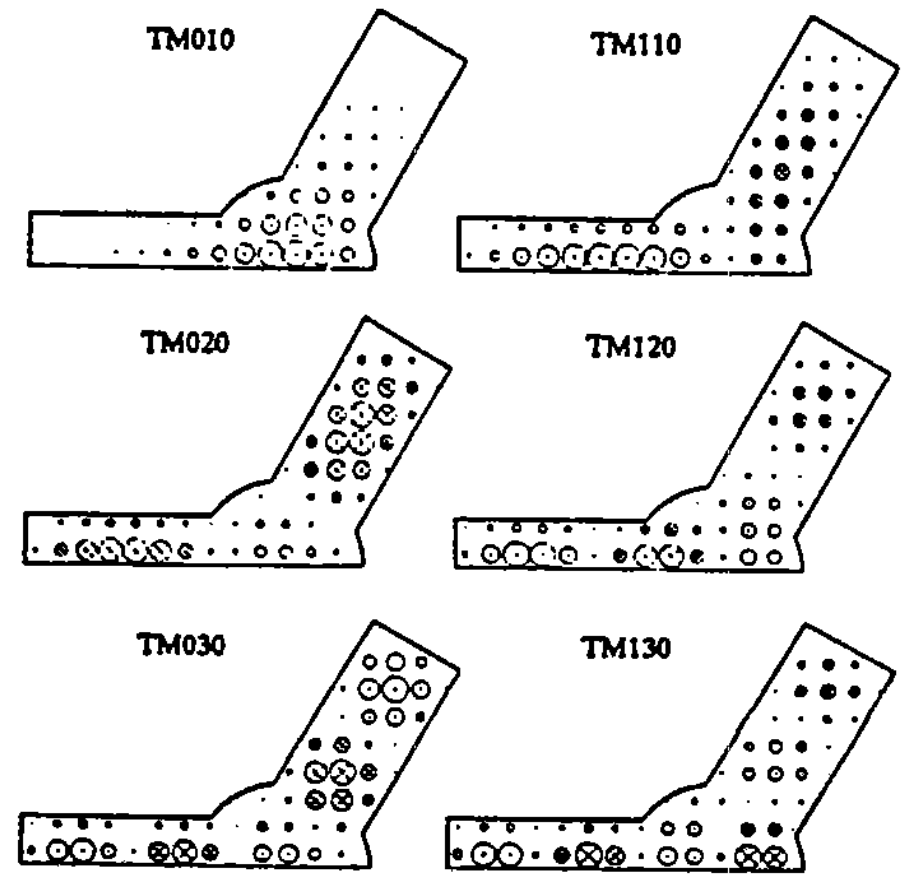

Fig. 2 Electric field plots from URMELT.T

A typical set of axial electric field plots is shown in Figure 2 for the $L=9.1 \mathrm{~cm}$ case. The phase shifts, delined as:

$$
\varphi=\frac{L}{c} \sqrt{\omega^{2}-\omega_{c}^{2}}+m \pi
$$

are plotted in Figure 3 for both the monopole (TMOn) and dipole (TMIn) modes as a function of frequency. In the above expression, $\omega$ is the angular frequency for a given mode, $\omega_{c}$ is the waveguide cutoff, $L_{f}$ is equal to $L-r$, and $c$ is the specd of light. The integet $m$ depends upon the branch on which the mode appears. When properly chosen all the points for a specific symmetry fall on a single curve. This is illustrated in Figure 3, which includes all data from four guide lengths (excluding the longest length). The phase shifts for the monopole modes (represented by data points on the upper curve) undergo a change less than $\pi$ in the frequency range considered. The data were fitted $10 \mathrm{a}$ single-resonance 4-point formula ${ }^{2}$ with a frequency of 9309 $\mathrm{MHz}$ and a $\mathrm{Q}$ of 2.3. The data for the dipole modes (lower curve) clearly show that there are two resonances in the same frequency range. The phase shifis in this frequency range undergo a change of $2 \pi$. The lower curve on Figure 3 is a theoretical fit obtained with a two-resonance form$u^{2}{ }^{2}$ for the phase shifts:

$$
\begin{gathered}
\varphi(\omega)=\tan ^{-1}\left(\frac{v_{1}}{\omega-u_{1}}\right)+\tan ^{-1}\left(\frac{v_{2}}{\omega-u_{2}}\right)-x(\omega)+m \pi \\
x(\omega)=x_{0}+x_{1} \omega
\end{gathered}
$$

The resonance frequencies of $4459 \mathrm{MHz}$ and $7350 \mathrm{Mhz}$ and their corresponding $Q$ of 2.7 and 6.3 were obtained from the six parameters $u_{1}, u_{2}, v_{1}, v_{2}, x_{0}$ and $x_{1}$ fitted to the data. A high degree of consistency for these results is evident by selecting data points from different crossed branches. We also used the data points from a single run with the longest guide length to calculate the dipole resonance frequency and $Q$. We obtained, using the fourpoint formula of reference 2, a resonance at $4311 \mathrm{MHz}$ with $Q=2.6$, or $4385 \mathrm{MHz}$ with $Q=2.9$, depending on the selection of the four points. These were consistent with the results for the lower resonance oblained from Figure 3 using data from four different runs. Data for the monopole modes using only the longest guide length were insufficient to yield a four-point solution. The extremely low $Q$ values for the single monopole and two dipole resonances lead us to conclude that principal TM mode resonances in the AC cavily are indeed highly damped.

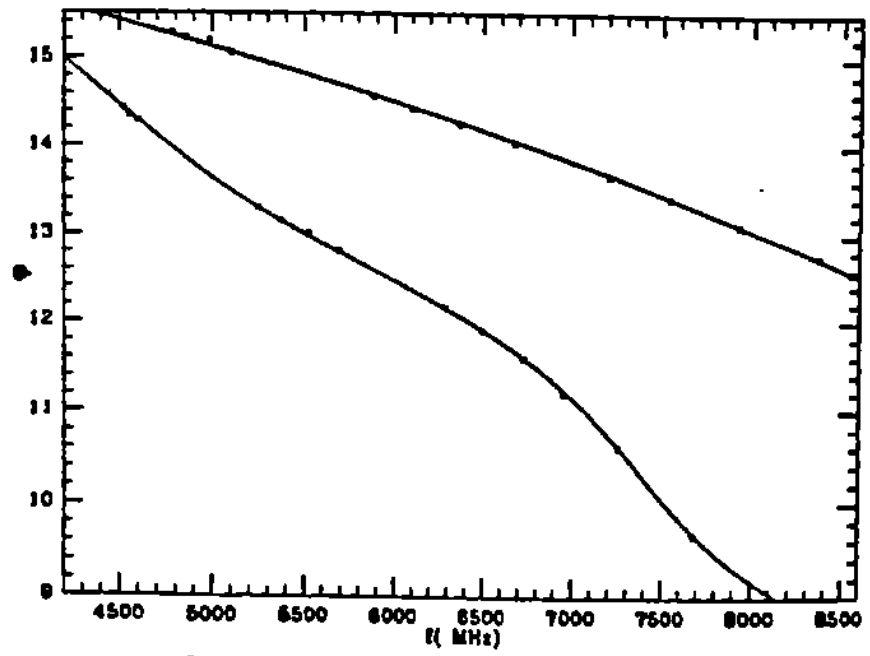

Fig. 3 Phasc shift vs frequency Plol 


\section{TRAPPED MODES AND DETRAPPING}

A review of the AC cavity URMEL-T plots of the electric lields in the plane perpendicular to the axis of the resonator shows that there are two possible trapped modes. These are shown in Figure 4, identified as (a) a TE111 mode, and (b) a TM011 mode in the cavity. To assess possiblc impact of thcse trapped modes on the accelerating cavity, we considered a $\pi$-mode, $X$-band accelerating structure with a threefold waveguide symmetry, similar to the AC cavity, using 3-D MAFIA models with up to 460000 mesh points. The 3-D models included long waveguide lengths (up to five times the cavity radius) and a finite cavily and waveguide height $(-\lambda$, where $\lambda$ is the fundamental wavelength). The waveguide width was chosen so that the cutoff frequency is above the fundamental and below the HOM. The MAFIA models confirm that the TE111 and TMO11 modes are in fact trapped in the cavity. The TE111 mode may not require damping because its impedance arises only from the probably very small TM contamination. On the other hand, damping is required for the TM011 mode. In order to minimize emittance growth of electron bunches over a very long distance, as in a linear collider, it was desirable to eliminate as much strayed

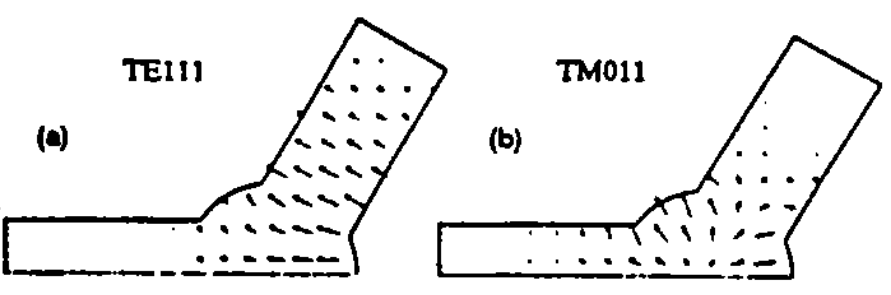

Fig. 4 Trapped modes in the AC cavily

fields in the structure as possible. We therefore considered methods to detrap the TE111 and TM011 modes. In a first unsuccessful attempt we tried to shift each of the three waveguides by a small oflsct (about $1 / 10$ of the cavily height) with respect to the cavily along the beam direction. This proved insufficient to detrap the modes. In a second atcompl, we tried to break the symmetry of the waveguide with respect to the cavity by reducing half of its height. This detrapped the TM011 mode (though not the TE111 mode), but it also distorted the accelerating mode? ${ }^{7}$. In a third attempt we increased the waveguide height by $50 \%$ in an unsymmetrical way with respect to the cavity. The results of this calculation are shown in Figures $5 a$ and $5 b$, showing two views of the detrapped TE111 and the TM011 mode, respectively. By making two MAFLA runs at different guide lengths, we were able to use the derivative method ${ }^{3}$ to calculate the loaded $Q$. The TEll1 mode is effectively detrapped with a $Q$ of 10.9 . The $Q$ of the TM011 mode is substantially reduced, but still over 100 . Oversized waveguides preclude installation on adjoining cavities. But damping waveguides may not be needed in every cavity if they are used in combination with other methods ${ }^{8}$ of wakefield suppression.
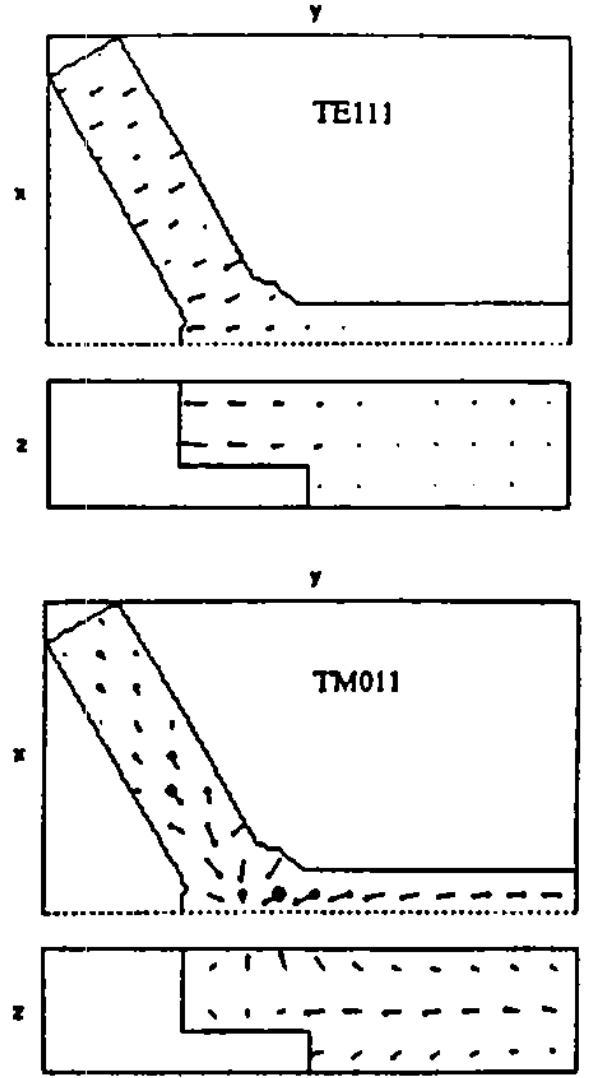

Fig. 5 Detrapped TE111 and TM011 modes

\section{CONCLUSIONS}

The problems of trapped modes which have persisted in previous damped structure designs have been partially solved with a threefold symmetry. Some progress has been made in detrapping the newly found trapped TM011 and TE111 modes unique to this geometry.

\section{Supported by DOE SBIR Grant No. DE-FG03-90ER81080. \\ tSupported by DOE Contracts DE-AC03-89ER40527 and DE-AC03. 76SFO0815.}

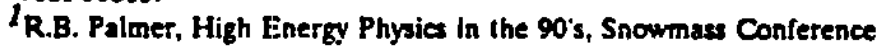
Proceedings, p. 638 (1989).

$2_{N}$. Kroll and D. Yu, Particle Acceleralors, 34, 231 (1990).

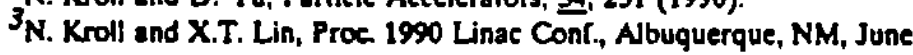
10.14 (1990) p. 238.

D. YU and Kroll, poster paper in Linear Acceleralor Codes Conf., Los Namos, NM, January 22-25 (1990); H. Deruyter, et al., Proc. 1990 Linac Conf., Albuquerque, NM, June 10-14 (1990) p. 132.

5. Goren and D. YU, Proc. 1989 IEEE Part. Acce. Conf., Chicago, IL, March 20.23 (1989) p. 189.

${ }^{6} \mathrm{G}$. Conciauro and P. Arcioni, European Particle Acceleralor Conference. Nice, France (1990).

${ }^{7} \mathbf{K}$ Ko has provided us with ARGUS compulations for a similar model considered lor this case, with an asymmetric step which reduces the waveguide height by $38 \%$. Using the derivalive method of reference 3 , we calculate $\mathrm{Q}$ of 74 for the TMO11 mode. The TM110 continues to exhibil exiremcly low $O$ 's.

${ }^{8} \mathrm{D}$. Yu and J.S. Kim, "Wakeficld Suppression Using Beatwave Cavities", this conference. 